\title{
The use of quadratic filter for the estimation of time-varying $\beta$
}

\author{
M. Gastaldi ${ }^{1}$, A. Germani ${ }^{1,2}$ \& A. Nardecchia ${ }^{1}$ \\ ${ }^{I}$ Department of Electrical and Information Engineering, University of \\ L'Aquila, Monteluco di Roio, L'Aquila, Italy \\ ${ }^{2}$ Istituto di Analisi dei Sistemi ed Informatica, CNR, Roma, Italy
}

\begin{abstract}
The beta parameter is used in finance to estimate systematic risk and usually it is assumed to be time invariant. The literature shows that there is now considerable evidence that beta risk is not constant over time. The aim of this paper is the estimation of time-varying Italian industry parameter betas using a new approach based on the Kalman filter technique and on polynomial estimates. This approach is applied to returns of the Italian market over the period 1991-2001. Keywords: time-varying beta, additive non-Gaussian noise, Kalman filter.
\end{abstract}

\section{Introduction}

The market effect on the returns of single assets is one of the most investigated arguments in finance. The Capital Asset Pricing Model (CAPM) suggests that the market effect is due to the relationship between the asset returns and the market portfolio returns. Moreover, the asset sensibility to the variations of the market portfolio returns produces the single asset expected returns. Parameter $\beta$ measures the asset sensibility to the variations on the market returns [1].

In the classical financial analysis, parameter $\beta$ is assumed to be time invariant and returns have a Gaussian distribution [2], but there is considerable general evidence that these assumptions are invalid in several financial markets as US markets [3] and Australia [4].

During the first 1970's researchers saw the first applications of the Kalman filter to the estimation of the systematic risk $[5,6]$. The proposed model for $\beta$ was the Random Walk Model [7] requiring the estimation of the unknown variances. Many researchers investigated the validity of the CAPM in presence of 
higher moments and their effects on asset prices. In [8] the $C A P M$ was extended to incorporate the effect of skewness on the asset evaluation, while in [9] the effect of co-curtosis on the asset prices was examined.

In this work we suppose that the asset systematic risk $\beta$ is time-variant nonGaussian and we study the Italian financial market describing the relation between the assets return and the market index return by means of the market model. We assume that $\beta$ follows a Random Walk Model. Starting from [10], where we supposed that random variables were Gaussian, we develop a new approach removing such hypothesis and we analyse a more realistic model where the random variables involved are non-Gaussian; since the knowledge of the asset return components is not complete, we assume that the moments of the random variables are unknown. Before starting with the estimation of $\beta$ we need to estimate such moments, by means of a Markov estimate [11].

As already mentioned, $\beta$ is non-Gaussian, therefore only the mean value and the variance of returns are not sufficient for the statistical characterization of the return distribution. In fact, it is known that in the Gaussian case the conditional expectation, which gives the minimum variance estimate, is a linear function of the observations and can be easily computed. In the non-Gaussian case this is not true, so that it is necessary to look for suboptimal estimates.

Following a state-space approach and adopting the minimum variance criterion [12], our aim is to find a more accurate estimate than the simple recursive linear one, that, as well known, admits the geometrical representation as the projection of the random $\beta$ in the Hilbert space of the linear transformation of the output, namely $\mathrm{L}(y)$. To improve such estimate our idea is to project it on the larger Hilbert space generated by the 2-nd order polynomial transformations of the output measurements, $\mathrm{P}(y)$. Because $\mathrm{P}(y)$ contains $\mathrm{L}(y)$ the estimation error will decrease. Our approach requires the definition of an "extended system", in which the output is defined as the aggregate of the original output and of its second order Kronecker powers.

This paper is organised as follows. In section 2 the standard market model regression able to define an unconditional beta for any asset is presented whereas in section 3 Kalman methodology, applied to the "extended system" by which conditional time dependent betas may be estimated, is analysed. Section 4 is devoted to present time-varying betas generated for Italian data and finally section 5 presents some conclusions based on the empirical evidence obtained in this study.

\section{The model}

The relation between the asset return and the market index return can be expressed as follows:

$$
R_{i, t}=\alpha_{i, t}+\beta_{i, t} R_{M, t}+\varepsilon_{i, t} \quad t=1, \ldots, T
$$

where:

- $\quad R_{i, t}$ is the return for the asset $i$ during the period $t$; 
- $R_{M, t}$ is the return for the market index during the period $t$;

- $\alpha_{l, t}$ is a random variable that describe the component of the return for the asset $i$ which is independent from the market return;

- $\varepsilon_{i, t}$ is the random disturbance vector such that:

$$
\begin{array}{llc}
\circ & E\left(\varepsilon_{i, t}\right)=0 ; & \forall i, \forall t \\
\circ & E\left(\varepsilon_{i, t} \varepsilon_{j, t}^{T}\right)=0 ; & \forall i, \forall j, \forall t, i \neq j \\
\circ & E\left(\varepsilon_{i, t} \varepsilon_{i, \tau}^{T}\right)=0 ; & \forall i, \forall t, \forall \tau, t \neq \tau \\
\circ & E\left(\varepsilon_{i, t} R_{M, t}^{T}\right)=0 . & \forall i, \forall t
\end{array}
$$

Equation (1) shows that the return for the asset $i$ during the period $t, R_{i, t}$, depends on the return for the market index $R_{M, t}$ on the same time. Moreover, the relation between these two variables is linear.

Coefficient $\beta$ is the most important parameter; it shows how asset returns vary with the market returns and is used to measure the asset systematic risk, or market risk.

\subsection{Random Walk model: hypothesis for our work}

In literature there are many models able to describe systematic risk. All of them can be represented by a simple two equation model. There are numerous studies assuming that asset prices follow the Random Walk model (RW) [7]. The Random Walk model can be expressed as follows

$$
\begin{aligned}
R_{i, t}= & \alpha_{i, t}+\beta_{i, t} R_{M, t}+\varepsilon_{i, t} \\
\alpha_{i, t} & =\alpha_{i, t-1}+u_{i, t} \\
\beta_{i, t} & =\beta_{i, t-1}+\eta_{i, t}
\end{aligned}
$$

We assume that the random variable $\beta_{0}$ (initial condition) and the random sequences $\left\{\varepsilon_{i, t}\right\},\left\{u_{i, t}\right\}$ and $\left\{\eta_{i, t}\right\}$ satisfy the following conditions for $\mathrm{t} \geq 0$ :

- $\mathrm{E}\left\{\varepsilon_{i, t}\right\}=0, \mathrm{E}\left\{u_{i, t}\right\}=0, \mathrm{E}\left\{\eta_{i, t}\right\}=0, \mathrm{E}\left\{\beta_{0}\right\}=0$;

- all the noises moments up to the 4 th order are finite;

- the noises $\left\{\varepsilon_{i, t}\right\},\left\{u_{i, t}\right\}$ and $\left\{\eta_{i, t}\right\}$ are the sequences of independent nonGaussian random variables.

We remark that no knowledge is assumed on the noises moments values; before proceeding is helpful to represent the Random Walk Model in the state space.

\subsection{System equations}

It is possible to define observation and state equations:

\section{- observation equation:}

$$
R_{i, t}=y(t)=C(t) x(t)+\psi(t)
$$

This equation represents the market model with time-varying coefficients.

Matrix $C(t)$ has dimensions $T \times 2$ so that each row will represent the observations at certain point in time; this matrix has the following structure

and is assumed to be known.

$$
C(t)=\left[1 \mid R_{M, t}\right]
$$

The state vector $x(t)$ has dimensions $2 \times 1$ and represents the $\alpha$ and $\beta$ 
coefficients at time $t$ :

$$
x(t)=\left[\alpha_{t} \mid \beta_{t}\right]^{T} .
$$

$\psi(t)$ is the part of the asset return $y(t)$ which is not modelled and represents the random sequence $\left\{\varepsilon_{l, t}\right\}$. The first four moments of the output noise are unknown, assumed finite and indicated by $E\left(\psi^{[h]}(t)\right), h=1, \ldots, 4$.

- $\quad$ state equation assumes this general form

$$
x(t)=A x(t-1)+\zeta(t)
$$

The first four moments of the state noise $\zeta(t)$ are assumed finite (for hypothesis), indicated by $E\left(\zeta^{\left[h^{\prime}\right]}(t)\right), h^{\prime}=1, \ldots, 4$ and its values are unknown.

In the model adopted in the present work (RW), matrix $A$ is the $2 \times 2$ identity matrix while vector $\zeta(t)$ models the random part of the state vector:

$$
\zeta(t)=\left[u_{t} \mid \eta_{t}\right]^{T} \text {. }
$$

Note that the values of the state noise moments depend on the moments of the random sequences $\left\{\eta_{i, t}\right\}$ and $\left\{u_{i, t}\right\}$, so that it is necessary to estimate six parameters - second, third and fourth moments of the sequences $\left\{\eta_{i, t}\right\},\left\{u_{i, t}\right\}$ (for hypothesis all the random sequences are zero mean).

Moreover we must estimate second, third and fourth moments for the three noise considered sequences. We represent these unknown parameters as a vector represented by $\vartheta=\left(\sigma_{u}^{2}, \sigma_{u}^{3}, \sigma_{u}^{4}, \sigma_{\eta}^{2}, \sigma_{\eta}^{3}, \sigma_{\eta}^{4}, \sigma_{\varepsilon}^{2}, \sigma_{\varepsilon}^{3}, \sigma_{\varepsilon}^{4}\right)$.

\section{The quadratic rilter and $\beta$ estimation}

As we have already seen in section 2, our aim is to find the minimum variance estimate of the state with respect to the output that coincides with its conditional expectation. While in the Gaussian case we obtain exactly a linear optimal solution, in our case the problem does not have an immediately recursive solution, so that we look for suboptimal estimates that are more accurate than the linear one.

To develop our approach, we need to use Kronecker algebra. Definitions and theorems that are necessary can be found in [13].

\subsection{The extended system}

To obtain the desired recursive estimates of (6) and (9) we define the 2-degree polynomial observation $\mathrm{Y} \in \mathfrak{R}^{\mu}, \mu=m+m^{2}$, where $m$ is the output dimension (in our case $m=1$ )

$$
\mathrm{Y}(t)=\left[\begin{array}{c}
y(t) \\
y^{[2]}(t)
\end{array}\right]
$$

and the extended state $\mathrm{X} \in \mathfrak{R}^{\chi}, \chi=n+n^{2}$, where $n$ is the state dimension (in our case $n=2$ ) 


$$
\mathrm{X}(t)=\left[\begin{array}{c}
x(t) \\
x^{[2]}(t)
\end{array}\right]
$$

where with $y^{[2]}(t)$ and $x^{[2]}(t)$ we denote, respectively, the 2 nd Kronecker power of the vectors $y$ and $x$.

We can now calculate the second Kronecker power of the state and the output equations

$$
\begin{gathered}
x^{[2]}(t)=A^{[2]}(t) x^{[2]}(t-1)+\zeta^{[2]}(t)+A(t) x(t-1) \otimes \zeta(t)+\zeta(t) \otimes A(t) x(t-1) \\
y^{[2]}(t)=C^{[2]}(t) x^{[2]}(t)+\psi^{[2]}(t)+C(t) x(t) \otimes \psi(t)+\psi(t) \otimes C(t) x(t)
\end{gathered}
$$

where with the symbol $\otimes$ we denote the Kronecker product.

By using some properties of the Kronecker algebra, it is possible to rewrite previous equations in a compact form and give the equations of the extended system

$$
\begin{aligned}
& \mathrm{X}(t)=\mathrm{AX}(t-1)+\mathrm{N}^{\prime}(t)+\mathrm{U}(t ; \vartheta) \\
& \mathrm{Y}(t)=\mathrm{C}(t) \mathrm{X}(t)+\mathrm{N}^{\prime \prime}(t)+\mathrm{V}(t ; \vartheta)
\end{aligned}
$$

where:

$$
\begin{aligned}
& \mathrm{X}(t)=\left[\begin{array}{c}
x(t) \\
x^{[2]}(t)
\end{array}\right] \quad \mathrm{Y}(t)=\left[\begin{array}{c}
y(t) \\
y^{[2]}(t)
\end{array}\right] \quad \mathrm{A}=\left[\begin{array}{cc}
A & 0 \\
0 & A^{[2]}
\end{array}\right] \\
& \mathrm{C}(t)=\left[\begin{array}{cc}
C(t) & 0 \\
0 & C^{[2]}(t)
\end{array}\right] \quad \mathrm{U}(t ; \vartheta)=\left[\begin{array}{c}
0 \\
E\left(\zeta^{[2]}(t)\right)
\end{array}\right] \quad \mathrm{V}(t ; \vartheta)=\left[\begin{array}{c}
0 \\
\left.E\left(\psi^{[2]}(t)\right)\right]
\end{array}\right] \\
& \mathrm{N}^{\prime}(t)=\left[\begin{array}{c}
\zeta(t) \\
\mathrm{N}^{\prime \prime}(t)=\left[\left(\zeta^{[2]}(t)\right)+\left(I_{2}+C_{2,2}^{T}\right)[A x(t-1) \otimes \zeta(t)]\right.
\end{array}\right] \\
& \psi \psi(t)-E\left(\psi^{[2]}(t)\right)+2[C(t) x(t) \otimes \psi(t)]
\end{aligned}
$$

indicating the dependence of vectors $\mathrm{U}$ and $\mathrm{V}$ on $\theta$. Matrix $I_{\mathrm{n}}$ is the identity matrix of dimension $n \times n$ and matrix $C_{.,}^{T}$ is a commutation matrix [14].

We call system (15) augmented system. Its state and observation noises $\left(\mathrm{N}^{\prime}(t)\right.$ and $\mathrm{N}^{\prime \prime}(t)$ respectively) are zero mean uncorrelated sequences and are also mutually uncorrelated at different times. For these noises we are able to calculate their autocovariances (for the initial hypothesis their cross covariance is null). Interested reader can found their expressions in [14]. Hence, for the augmented system the optimal linear state estimate can be calculated by means of the Kalman filter equations.

\subsection{Quadratic filter}

In economic systems, the covariance matrices for the various noise processes in the model are assumed to be known and assigned a priori. In this paper we estimate the covariance matrices by means of the observations of the returns to individual assets and the market portfolio.

We can define the following cost index to be minimized in order to obtain the desired estimation 


$$
\begin{aligned}
& J(\vartheta)=\sum_{t=1}^{T}\left([\mathrm{Y}(t)-\mathrm{V}(t ; \vartheta)-\mathrm{C}(t)(\mathrm{AX}(t-1)+\mathrm{U}(t ; \vartheta))]^{T}[\mathrm{C}(t) P(t \mid t-1 ; \vartheta) .\right. \\
& \left.\left.\cdot \mathrm{C}^{T}(t)+R(t ; \vartheta)\right]^{T}[\mathrm{Y}(t)-\mathrm{V}(t ; \vartheta)-\mathrm{C}(t)(\mathrm{AX}(t-1)+\mathrm{U}(t ; \vartheta))]\right),
\end{aligned}
$$

where $\operatorname{Pp}(\mathrm{t} \mid t-1 ; \theta)$ is the prediction covariance and $\mathrm{R}(\mathrm{t} ; \theta)$ is the covariance of the output equivalent noise (16). The above function has been minimized by means of the Markov estimate [15]. When the estimation $\hat{\vartheta}$ of the parameter vector is calculated, the optimum estimation of the extended state vector is obtained by means of the Kalman filter, by using the system matrices evaluated for $\hat{\vartheta}$.

Using the obtained results and taking into account the deterministic and the stochastic input we can use the Kalman filter for the extended system.

The filter need to be initialised; initial conditions for the state vector and for the prediction covariance matrix are:

$$
\hat{\mathrm{X}}(0 \mid-1)=E\{\mathrm{X}(0)\}=0, P(0 \mid-1)=E\left\{\mathrm{X}(0) \mathrm{X}(0)^{T}\right\}=\Psi_{\mathrm{X}(0)}
$$

Afterwards, it is possible to proceed with the estimation algorithm. At each time $t$, following steps are reiterated:

$$
\begin{gathered}
P_{p}(t)=\mathrm{A} P(t-1) \mathrm{A}^{T}+\mathrm{Q}(t ; \hat{\vartheta}) \\
K(t)=P(t \mid t-1) \mathrm{C}^{T}(t)\left(\mathrm{C}(t) P(t \mid t-1) \mathrm{C}^{T}(t)+\mathrm{R}(t ; \hat{\vartheta})\right)^{-1} \\
P(t)=[I-K(t) \mathrm{C}(t)] P(t \mid t-1) \\
\hat{\mathrm{X}}(t \mid t-1)=\mathrm{A} \hat{\mathrm{X}}(t-1)+\mathrm{U}(t ; \hat{\vartheta}) \\
\hat{\mathrm{X}}(t)=\hat{\mathrm{X}}(t \mid t-1)+K(t)(\mathrm{Y}(t)-\mathrm{C}(t) \hat{\mathrm{X}}(t \mid t-1))
\end{gathered}
$$

where $K(t)$ is the filter gain, $P(t)$ and $P(t \mid t-1)$ are respectively the filter and prediction covariances.

The optimal linear estimate of the augmented state process $\mathrm{X}(k)$ with respect to the augmented observations $Y(k)$ agrees with its optimal quadratic estimate with respect to the original observations $y(k)$, in the sense of taking into account the second power of $y(k)$. We obtain in this way the optimal quadratic estimate of the system (6) and (9). The optimal linear estimate of the original state $x(k)$ with respect to the same sets of augmented observations is easily determined by extracting the first $n$ components in the vector $\hat{X}(k)$ (recall that in our case $n=2)$.

The optimal estimate of parameter at each time $t$ is then determined by extracting the second component in the vector $\hat{x}(t)$.

We stress that the proposed algorithm, if we do not calculate the second power of the observations, produces the best linear filter, which coincides, as is well known, with the optimal filter when the noises are Gaussian. Consequently, it becomes necessary to consider higher order filters when the noises have distribution far from the Gaussian. By observing formulas that define the augmented system parameters, it becomes evident that the computational effort of the polynomial filter quickly grows with increasing filter order. However, we point out that even low-order polynomial filters (the quadratic filter considered in our case) which do not require a particular sophisticated implementation, show very high performances with respect to the linear filter. 


\subsection{Goodness of the proposed method}

We assess the accuracy of the forecast using the MAE (Mean Absolute Forecasting Error indices) and MSE (Mean Square Forecasting Error) indices [16]:

1. Mean Absolute Forecasting Error: once we forecast $\hat{R}_{i t}$ it is possible to measure estimation accuracy using a measure of forecast error which compares the forecast to actual values by

$$
M A E_{i}=\sum_{t=1}^{T} \frac{\left|\hat{R}_{i t}-R_{i t}\right|}{T}
$$

A potential problem with the use of MAE measure is that all errors have the same weight. An alternative approach is to give an heavier penalty on outliers then the MAE measure with the use of squared term by the following index:

2. Mean Square Forecasting Error (MSE):

$$
M S E_{i}=\sum_{t=1}^{T} \frac{\left(\hat{R}_{i t}-R_{i t}\right)^{2}}{T}
$$

Table 1: $\quad$ Statistics for weekly returns data.

\begin{tabular}{|l|r|r|r|r|}
\hline \multicolumn{1}{|c|}{ ISX Industry } & \multicolumn{1}{c|}{ Mean } & Standard Deviation & \multicolumn{1}{c|}{ Skewness } & \multicolumn{1}{c|}{ Kurtosis } \\
\hline Food (7) & 0.0973 & 3.9622 & 7.0161 & 108.0010 \\
Insurance (19) & 0.1936 & 3.4726 & 0.6320 & 5.4050 \\
Transport (13) & 0.2253 & 2.8966 & 0.3902 & 5.0322 \\
Banks (53) & 0.2647 & 2.6466 & 0.8518 & 7.2513 \\
Paper (2) & -0.0041 & 4.3221 & 0.9565 & 6.3610 \\
Chemicals (21) & 0.2173 & 2.5915 & 0.7134 & 4.9909 \\
Building materials (13) & 0.1973 & 3.2434 & 0.5810 & 4.2747 \\
Distribution (6) & 0.3348 & 3.5637 & 0.5201 & 4.7679 \\
Publishing (11) & 0.3351 & 3.8691 & 1.5298 & 11.6685 \\
Electronics (29) & 0.1712 & 2.5269 & 0.6609 & 5.4241 \\
Diversified financials (4) & 0.3863 & 4.7617 & 4.0280 & 34.2872 \\
Financial holdings (29) & 0.1610 & 3.2995 & 0.5838 & 4.5906 \\
Real estate (21) & 0.2525 & 3.3365 & 1.2264 & 7.2478 \\
Equipments (9) & 0.3017 & 3.0579 & 0.6262 & 5.4661 \\
Miscellaneous industries (2) & 0.1313 & 5.6184 & 0.1467 & 11.4537 \\
Minerals (7) & 0.2209 & 3.0296 & 0.7040 & 6.1690 \\
Public utility (18) & 0.3482 & 2.5856 & 0.4932 & 3.5527 \\
Financial services (3) & 0.0577 & 3.6974 & 0.6715 & 4.8213 \\
Textile (27) & 0.2305 & 2.9455 & 5.5834 & 77.8821 \\
Tourism and leisure (14) & 0.3072 & 3.0166 & 1.0332 & 6.0254 \\
Market Index & 0.2198 & 2.1500 & 0.5214 & 4.7846 \\
\hline
\end{tabular}

\section{Empirical results}

The concept of beta is well known in the financial community and its values are estimated by various technical service organizations.

Generally speaking, we expect that aggressive companies or highly leveraged companies have high betas, whereas companies whose performance is unrelated 
to the general market behaviour have low betas. In this paper the data used are weekly price relative information for 20 Italian Stock Exchange industries provided by TraderLink s.r.1.. Our full sample period is extended from May 1991 to June 2001. The data were expressed in Italian lyres and percentage returns were created for the analysis.

In table 1 are reported information about the distributional properties of the industry sector returns used in our study; in the first column the name of the industry and the number of considered firms in each sector (in parenthesis) are reported. Note that there is a correlation between the risk of each industry (the standard deviation) and the number of firms in each sector. In fact, the standard deviation for the industry with the largest number of firms (Banks 53 companies) has a smaller value than the Paper industry ( 2 firms). Distribution of the industry return is leptokurtic. Moreover Diversified financials, Food and Textile exhibit high level of skewness.

Table 2: $\quad$ MAE and MSE forecast error results.

\begin{tabular}{|l|c|c|r|r|r|r|}
\hline \multirow{2}{*}{\multicolumn{1}{|c|}{ ISX industry }} & \multicolumn{3}{|c|}{ MAE } & \multicolumn{3}{c|}{ MSE } \\
\cline { 2 - 7 } & $\begin{array}{c}\text { Linear } \\
\text { Filter }\end{array}$ & $\begin{array}{c}\text { Quadratic } \\
\text { Filter }\end{array}$ & $\begin{array}{c}\text { Improvement } \\
\left(\mid \text { MAE }_{\mathrm{Q}}-\mathrm{MAE}_{\mathrm{L}}\right)\end{array}$ & $\begin{array}{c}\text { Linear } \\
\text { Filter }\end{array}$ & $\begin{array}{c}\text { Quadratic } \\
\text { Filter }\end{array}$ & $\begin{array}{c}\text { Improvement } \\
\left(\left|\mathrm{MAE}_{\mathrm{Q}}-\mathrm{MAE}_{\mathrm{L}}\right|\right)\end{array}$ \\
\hline Food & 0.8061 & $1.7020 \mathrm{e}-2$ & 0.7891 & 1.3679 & $6.1831 \mathrm{e}-4$ & 1.3673 \\
Insurance & 0.7073 & $1.4641 \mathrm{e}-2$ & 0.6926 & 0.9412 & $4.1161 \mathrm{e}-4$ & 0.9408 \\
Transport & 0.5907 & $1.2154 \mathrm{e}-2$ & 0.5785 & 0.6453 & $2.7302 \mathrm{e}-4$ & 0.6450 \\
Banks & 0.4515 & $9.1051 \mathrm{e}-3$ & 0.4424 & 0.3894 & $1.6032 \mathrm{e}-4$ & 0.3892 \\
Paper & 1.2874 & $2.5247 \mathrm{e}-2$ & 1.2622 & 3.2974 & $1.2571 \mathrm{e}-3$ & 3.2961 \\
Chemicals & 0.4741 & $9.9739 \mathrm{e}-3$ & 0.4641 & 0.4217 & $1.8257 \mathrm{e}-4$ & 0.4215 \\
Building materials & 0.6840 & $1.4281 \mathrm{e}-2$ & 0.6697 & 0.9303 & $4.2506 \mathrm{e}-4$ & 0.9299 \\
Distribution & 0.8611 & $1.8579 \mathrm{e}-2$ & 0.8425 & 1.3955 & $6.6215 \mathrm{e}-4$ & 1.3948 \\
Publishing & 0.9296 & $1.8035 \mathrm{e}-2$ & 0.9116 & 1.8858 & $6.9256 \mathrm{e}-4$ & 1.8851 \\
Electronics & 0.4771 & $9.3423 \mathrm{e}-3$ & 0.4677 & 0.4118 & $1.6036 \mathrm{e}-4$ & 0.4116 \\
Diversified financials & 1.1486 & $2.1778 \mathrm{e}-2$ & 1.1268 & 3.4293 & $1.2267 \mathrm{e}-3$ & 3.4281 \\
Financial holdings & 0.5255 & $1.0353 \mathrm{e}-2$ & 0.5151 & 0.4930 & $1.9494 \mathrm{e}-4$ & 0.4928 \\
Real estate & 0.7240 & $1.3892 \mathrm{e}-2$ & 0.7101 & 1.1918 & $4.4448 \mathrm{e}-4$ & 1.1914 \\
Equipments & 0.7728 & $1.5111 \mathrm{e}-2$ & 0.7577 & 1.1851 & $4.5278 \mathrm{e}-4$ & 1.1846 \\
Misc. industries & 1.6283 & $3.2820 \mathrm{e}-2$ & 1.5955 & 6.2683 & $2.4329 \mathrm{e}-3$ & 6.2659 \\
Mineral & 0.7874 & $1.5627 \mathrm{e}-2$ & 0.7718 & 1.2061 & $4.9693 \mathrm{e}-4$ & 1.2056 \\
Public utilities & 0.6433 & $7.2584 \mathrm{e}-3$ & 0.6360 & 0.7298 & $9.7258 \mathrm{e}-5$ & 0.7297 \\
Financial services & 1.0708 & $2.1248 \mathrm{e}-2$ & 1.0495 & 2.1797 & $8.8923 \mathrm{e}-4$ & 2.1788 \\
Textiles & 0.5191 & $9.9448 \mathrm{e}-3$ & 0.5091 & 0.4816 & $1.7282 \mathrm{e}-4$ & 0.4814 \\
Tourism and leisure & 0.6969 & $1.3613 \mathrm{e}-2$ & 0.6833 & 1.0309 & $3.8915 \mathrm{e}-4$ & 1.0305 \\
\hline
\end{tabular}

The standard market model was estimated for every Italian industry, using the domestic market index. To evaluate the performance of beta estimates we calculate the MAE and MSE metrics presented above ((23)-(24)). The MSE and MAE measures are presented in table 2.

Notice that the proposed method (the quadratic filter) produced in all 20 industries low level of forecast error demonstrating the effectiveness of the chosen estimation approach.

It is important to emphasize that quadratic filter follows variations of $\beta$ parameter better than the linear one, so that the output restored by means of the estimated parameters in the case of quadratic filter is more similar to the true output than the output obtained by means of the linear filter, as shown in the 
following figures 1 and 2 .

In these figures is represented a comparison between a portion of the true output (returns for the Public utilities sector) and the restored output so that it is possible to better appreciate the performances of the two filters. It is evident that in the quadratic case the restored output practically coincides with the true output.

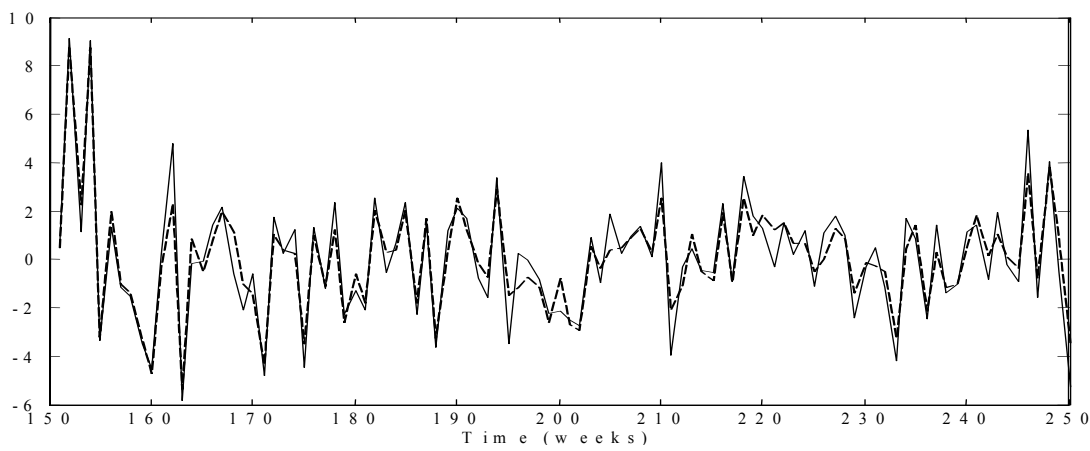

Figure 1: $\quad$ Matching between true and restored output (Linear filter).

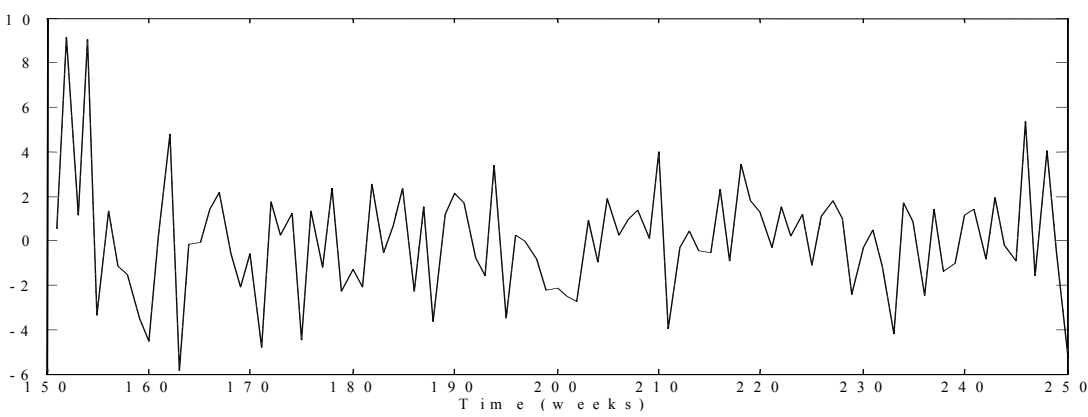

Figure 2: $\quad$ Matching between true and restored output (Quadratic filter).

\section{Conclusions}

In this paper we face the problem of systematic risk beta estimation. The presented results show that it is possible to estimate conditional time-dependent betas applying the quadratic filter to a sample of returns on Italian industry portfolios over the period 1991-2001. The obtained results by the proposed method are indeed much more accurate than those obtained by the classical linear filtering. 


\section{References}

[1] R.J. Fuller and J.L. Farrell, Analisi degli investimenti finanziari, McGrawHill: Milano; 1993.

[2] E.F. Fama, "Risk, return and equilibrium: some clarifying comments", Journal of Finance, vol.23 n.1, 1968, pp 29-40.

[3] F.J. Fabozzi and J.C. Francis, "Beta as a random coefficient", Journal of Financial and Quantitative Analysis, vol. 13, 1978, pp 101-115.

[4] R.W Faff, J.H.H. Lee and T.R.L. Fry, "Time stationarity of systematic risk: Some Australian evidence", Journal of Business Finance and Accounting, vol. 19, 1992, pp 253-270.

[5] M. Kantor, "Market Sensitivities", Financial Analysts Journal, vol.27 n.1, 1971, pp 64-68.

[6] K. Garbade and J. Rentzler, "Testing the hypothesis of beta stationarity", International Economic Review, vol. 22 n.3, 1981, pp 577-587.

[7] C. Wells, The Kalman Filter in Finance, Kluwert Academic Publishers: Dordrecht; 1996.

[8] R.S. Sears and K.C.J. Wei, "Asset Pricing, Higher Moments, and the Market Risk Premium: a note”, Journal of Finance, vol. 40, 1985, pp 1251-1253.

[9] H. Fang and T-Y. Lai, "C-Kurtosis and Capital Asset Pricing", The Financial Review, vol. 32 n.2, 1997, pp 293-307.

[10] M. Gastaldi and A. Nardecchia, "The Kalman filter approach for timevarying $\beta$ estimation", System Analysis Modelling Simulation, vol.43 n.8, 2003, pp 1033-1042.

[11] L. Lyung, System identification - theory for the user, New York: Prentice Hall; 1987.

[12] F. Carravetta, A. Germani and M. Raimondi, "Polynomial Filtering for Linear discrete time non-Gaussian systems", SIAM J.Control Optim., vol.34 n.5, 1996, pp 1666-1690.

[13] R. Bellman, Introduction to Matrix Analysis. New-York: McGraw-Hill; 1970.

[14] M. Dalla Mora, A. Germani and A. Nardecchia, "Restoration of Images Corrupted by Additive non-Gaussian Noise", IEEE Trans. on Circuits and Systems I: Fundamental Theory and Applications, vol.48 n.7, 2001, pp 859-875.

[15] A.V. Balakrishnan, Kalman Filtering Theory. New York: Optimization Software, Inc., Publication Division; 1984.

[16] M.D. McKenzie, R.D. Brooks and R.W. Faff, "The use of domestic and world market indexes in the estimation of the time-varying betas", $J$. of Multinational Financial Management, vol.10, 2000, pp 91-106. 